\title{
Cómo trabajar la orientación espacial de modo significativo en Educación Infantil: implicaciones didácticas
}

\section{How to develop spatial orientation skill in a meaningful context in Early Childhood Education: didactical implications.}

\section{Clara Jiménez-Gestal ${ }^{1}$ Ainhoa Berciano ${ }^{2}$ \\ María Salgado ${ }^{3}$}

\begin{abstract}
Resumen: En este trabajo presentamos una práctica docente distinta en Educación Infantil que favorece el desarrollo de la capacidad espacial temprana y, en especial, las habilidades relacionadas con tareas de orientación espacial. Para tal fin, se ha diseñado una secuencia de actividades basadas en la Enseñanza Matemática Realista que pueda ser implementada en el aula de Educación Infantil con niños y niñas de edades comprendidas entre los 3 y los 6 años. Además del diseño e implementación, se muestran las peculiaridades de implementación en cada curso y algunas producciones realizadas por los niños y niñas dependiendo de la edad y de su aprendizaje con el fin de ver los avances en cada caso.
\end{abstract}

Palabras clave: Educación Infantil, orientación espacial, Enseñanza Matemática Realista, práctica docente

Fecha de recepción: 26 de noviembre de 2018. Fecha de aceptación: 12 de febrero de 2019.

1 Universidad de La Rioja, clara.jimenez@unirioja.es, orcid.org/0000-0003-1766-2855

2 Universidad del País Vasco/ Euskal Herriko Unibertsitatea, ainhoa.berciano@ehu.eus, orcid.org/00000001-7399-4745

3 Universidad de Santiago de Compostela, maria.salgado@usc.es, orcid.org/0000-0002-0309-241X 


\begin{abstract}
In this paper we present a different teaching practice in Early Childhood Education that favours the development of early spatial capacity and, especially, skills related to spatial orientation tasks. To this end, a sequence of activities based on Realistic Mathematical Education has been designed that can be implemented in Early Childhood Education classroom with children between the ages of 3 and 6 . In addition to the design and implementation, we show the peculiarities of implementation in each course and some productions made by children depending on age and their learning in order to see the progress in each case.
\end{abstract}

Keywords: Early Childhood Education, Spatial Orientation, Realistic Mathematical Education, Teaching Practice

\title{
INTRODUCCIÓN Y MARCO TEÓRICO
}

A pesar de que es en la primera infancia donde se asientan los conocimientos matemáticos que se usarán en el futuro, el tratamiento de las matemáticas es en muchas ocasiones difuso y la adquisición de competencias es difícil de evaluar. En este sentido, como advierte Rico (1993), es muy raro que se enfrente a los niños a actividades creativas en las que sean evaluadas las competencias que se desarrollan para resolverlas y, aunque los niños poseen muchas capacidades matemáticas cuando se escolarizan, para la gran mayoría las matemáticas escolares son difíciles y confusas. Para colmo, algunos profesores afirman que se pierde mucho tiempo rellenando fichas, repitiendo mecanismos, y ejercitando destrezas. Sin embargo, como apuntan las directrices curriculares y tendencias metodológicas actuales (MEC, 2007), el pensamiento matemático no se aprende recibiendo información de forma pasiva o aprendiendo unas técnicas sino a través de un proceso activo y un deseo de hallar una respuesta a contextos y problemas reales.

Por este motivo, teniendo en cuenta que las niñas y los niños adquieren diversos conocimientos informales en su entorno vital, convendría entender cómo realizan el aprendizaje matemático y, en particular, el espacial.

Con el fin de ahondar en este objetivo, debemos destacar que las matemáticas en Educación Infantil, y en particular sus metodologías, han evolucionado en los últimos tiempos tratando de dar respuesta a preguntas candentes 
relacionadas con nuestro objetivo, pero que siguen sin tener una solución universal que facilite la enseñanza-aprendizaje.

Dentro del apartado referido a las metodologías de enseñanza-aprendizaje de la matemática cabe destacar la figura de Freudenthal, considerado el padre de la Matemática Realista. Alejándose de aprendizajes antiguos, memorísticos, abstractos, y en los cuales el profesorado se limita a dar sus lecciones y a corregir las pruebas escritas, la Matemática Realista propone buscar en una fase inicial contextos reales para una construcción significativa del aprendizaje matemático.

En concreto, se basa en la idea de que la matemática -si ha de tener valor humano- debe estar conectada con la realidad, mantenerse cercana a las niñas y los niños y ser relevante para la sociedad (Freudenthal, 1977). La idea que subyace a este enfoque es la afirmación de que aunque no todos los niños y niñas deban llegar a ser matemáticos o matemáticas en la edad adulta, sí todas las personas adultas deben usar las matemáticas para resolver los problemas de la vida cotidiana.

Recogiendo las exposiciones de Freudenthal, Alsina (2009) describe la EMR a partir de los siguientes principios:

1. De actividad: Las matemáticas concebidas como una actividad humana. La finalidad de las matemáticas es matematizar (organizar) el mundo que nos rodea.

2. De realidad: Las matemáticas se aprenden haciendo matemáticas en contextos reales o realistas.

3. De niveles: Los estudiantes pasan por distintos niveles de comprensión: situacional (en el contexto de la situación); referencial (esquematización a través de modelos, descripciones, etc.); general (exploración, reflexión y generalización); formal (procedimientos estándares y notación convencional).

4. De reinvención guiada: Proceso de aprendizaje que permite reconstruir el conocimiento matemático formal a través de la mediación. El docente observa el proceso de aprendizaje para poder diseñar las experiencias en las que se reinventa la matemática.

5. De interacción: La enseñanza de las matemáticas es considerada una actividad social. La interacción entre los estudiantes y entre los estudiantes y los profesores puede provocar que cada uno reflexione a partir de lo que aportan los demás y así poder alcanzar niveles más altos de comprensión. 
6. De interconexión: Los bloques de contenido matemático (numeración y cálculo, álgebra, geometría,... ) están relacionados entre sí, no pueden ser tratados como entidades separadas.

Los niños deben, pues, aprender matemáticas en contextos reales y cercanos que tengan para ellos sentido, a partir de los cuales desarrollar conceptos y aplicar reglas. Surge así la necesidad de la matematización: trasladar un problema de la vida cotidiana al mundo de las matemáticas, resolverlo, y volver a trasladarlo al mundo real, lo que familiariza al alumno con el mundo matemático.

Si volvemos a nuestro objetivo original, el de fomentar el aprendizaje matemático en edades tempranas y, en particular, la orientación espacial, en primer lugar debemos decir qué entendemos por ésta. En este sentido, Clements y Sarama (2009, p. 161) definen la orientación espacial:

...requiere entender y ser capaz de analizar y establecer las relaciones existentes entre distintas posiciones en el espacio; en primer lugar, con respecto a la posición de uno mismo y con respecto al movimiento, para, finalmente, ser capaz de trabajar desde perspectivas más abstractas que incluyen el tratamiento de mapas y uso de coordenadas con distintas escalas.

Aun así, es cierto que la capacidad relacionada con la orientación espacial es muy ambiciosa y debemos nuevamente focalizar nuestra atención en el aula de infantil. En este caso, el NCTM (2000, p.100), en la etapa Pre K-2, describe que "localizar y describir relaciones espaciales mediante coordenadas geométricas y otros sistemas de representación" debe capacitar al infante para que sea capaz de:

1. Describir, dar nombre e interpretar posiciones relativas en el espacio y aplicar ideas sobre posición relativa (c1);

2. Describir, dar nombre e interpretar la dirección y la distancia en los desplazamientos en el espacio y aplicar estas nociones (c2);

3. Encontrar y denominar "lugares" con relaciones simples como "cerca de" y en sistemas de coordenadas tales como mapas (c3).

Igualmente, dentro del apartado "utilizar la visualización, el razonamiento matemático y la modelización geométrica para resolver problemas", destacamos:

4. Reconocer formas y estructuras geométricas en el entorno, y determinar su situación (c4). 
Si bien, en Gonzato, Fernández Blanco y Díaz Godino (2011) se analizan tareas presentadas en diversas investigaciones sobre visualización y orientación espacial, se puede apreciar que la mayor parte de las mismas no hacen diferenciación por edades y se enfocan al currículo de educación primaria.

Es por ello que, si queremos analizar el dilema de la orientación espacial en edades tempranas, debemos destacar el trabajo de Berciano, Jiménez-Gestal y Salgado (2017) en el que analizan de modo cualitativo el tipo de representación simbólica que son capaces de realizar las niñas y niños de 5 años cuando se les plantea una actividad de orientación espacial en contexto, basada en la Enseñanza Matemática Realista. En concreto, concluyen que todos los niños y niñas participantes en la actividad entienden el concepto de 3-dimensionalidad y los cambios producidos en el eje vertical, lo que les lleva a usar diversos símbolos en sus representaciones y que, además, la complejidad de los símbolos depende de su capacidad espacial.

Dado que el currículo de educación infantil no diferencia por edad entre los 3 y los 6 años y vistos los resultados obtenidos con niñas y niños del último curso de Educación Infantil, se quería indagar por la idoneidad de la práctica docente en edades más tempranas, al igual que los posibles resultados y representaciones que son capaces de realizar los niños y niñas. Con esto se pretende, acorde a NCTM y directrices internacionales, trabajar del modo más adecuado, en contexto, la capacidad de orientación a lo largo de todo el ciclo.

\section{OBJETIVO}

El objetivo de la investigación es describir las características principales de una secuencia didáctica diseñada específicamente para trabajar la orientación espacial en el segundo ciclo de educación infantil. Para tal fin, distinguiremos los contenidos que se pueden tratar según la edad, describiremos las diferentes producciones realizadas por niñas y niños de distintas edades y detallaremos las ventajas y dificultades docentes asociadas a la implementación. 


\section{DISEÑO TEÓRICO}

El diseño de la actividad, en su estructura secuencial de actividades, es el mismo que aparece descrito en Berciano, Jiménez-Gestal y Salgado-Somoza (2016) y que resumimos a continuación: a los niños y niñas de las tres aulas con edades comprendidas entre los 3 y los 6 años se les plantea como contexto motivador que deben "encontrar el tesoro perdido y escondido en el Colegio".

Tal como se describe en Berciano et al. (2016), la actividad realizada en 5 años fue un éxito, por lo que en este artículo nos planteamos transferir dicha actividad, manteniendo su contexto, al aula de 3 y 4 años, y revisar las dificultades y ventajas que esta trasferencia puede suponer. Para ello, la actividad debe ser readaptada a cada curso, por lo que se debe garantizar que la matematización asociada sea la adecuada a la edad correspondiente (ver tabla 1).

\section{FASES DE LA EXPERIMENTACIÓN}

La experimentación en todas las edades ha constado de 3 fases perfectamente delimitadas y diferenciadas desde el punto de vista teórico:

1. Trabajo previo en el aula: se trabajan las ideas previas relativas a los piratas, su modo de vida, los mapas, representaciones gráficas, etc. En esta fase, se realizan preguntas abiertas para que todas/os puedan participar. Se consensuan algunas ideas al igual que la búsqueda del tesoro, definiendo estrategias de búsqueda y tipos de agrupaciones.

2. Trabajo en contexto: se lleva a cabo la búsqueda del tesoro (consistente en una bolsa de monedas de chocolate) en pequeños grupos.

3. Trabajo posterior en el aula: se lleva a cabo una representación verbal y una representación simbólica a través de los dibujos. Individualmente deben hacer un plano del recorrido realizado y describir verbalmente su recorrido, el significado de los símbolos usados en el mismo, etc.

Con el tesoro, en cada grupo de estudiantes, atendiendo a la dificultad según la edad, tendrán que clasificar, ordenar, cuantificar y registrar el "dinero" que han encontrado. 


\section{CONTENIDOS ESPECÍFICOS A CADA EDAD}

Matematización asociada a todas las edades del ciclo de educación infantil:

1. Contenidos c1, c2, c3, c4 (detallados en el apartado de estándares de contenidos de la NCTM).

2. Competencia comunicativa.

3. Números y cantidad.

4. Formas geométricas y sus propiedades.

5. Clasificación y reconocimiento de significados de pictogramas como acciones cotidianas.

Para poder adaptar a cada curso la actividad, la maestra ha hecho una reflexión previa acerca de los contenidos que se pueden trabajar en cada edad y mediante conversaciones con las niñas y niños de cada clase ha trabajado los contenidos que aparecen descritos a continuación.

Tabla 1. Contenidos que se trabajan en cada edad

\begin{tabular}{llll}
\hline \multicolumn{1}{c}{ Contenidos/Edad } & \multicolumn{1}{c}{ Edad 3 años } & \multicolumn{1}{c}{ Edad 4 años } & \multicolumn{1}{c}{ Edad 5 años } \\
\hline Posiciones relativas (c1) & $\begin{array}{l}\text { Arriba/abajo; dentro/ } \\
\text { fuera }\end{array}$ & A un lado/ al otro & $\begin{array}{l}\text { lzquierda/ derecha; } \\
\text { en frente de }\end{array}$ \\
\hline $\begin{array}{l}\text { Dirección y distancia } \\
\text { (c2) }\end{array}$ & $\begin{array}{l}\text { Hacia delante/ hacia } \\
\text { atrás; cerca/lejos }\end{array}$ & $\begin{array}{l}\text { Más cerca que/ más } \\
\text { lejos que }\end{array}$ & $\begin{array}{l}\text { Estimación de } \\
\text { distancias: pasos }\end{array}$ \\
\hline $\begin{array}{l}\text { Sistemas de } \\
\text { coordenadas (c3) }\end{array}$ & $\begin{array}{l}\text { Ubicación del punto } \\
\text { de interés como punto } \\
\text { absoluto en el mapa }\end{array}$ & $\begin{array}{l}\text { Aparición de elementos } \\
\text { de referencia } \\
\text { singulares }\end{array}$ & $\begin{array}{l}\text { Representación de } \\
\text { puntos de } \\
\text { referencia locales }\end{array}$ \\
\hline Formas y situación (c4) & $\begin{array}{l}\text { Formas planas } \\
\text { Formas planas }\end{array}$ & $\begin{array}{l}\text { Cuerpos } \\
\text { geométricos } \\
\text { notables }\end{array}$ \\
\hline $\begin{array}{l}\text { Competencia } \\
\text { comunicativa }\end{array}$ & $X$ & $X$ & $X$ \\
\hline Números y cantidad & Muchos/pocos & Identificación de & Cardinales y \\
cardinales & ordinales \\
\hline Formas geométricas & Objetos 2D & Objetos 2D & Objetos 2D y 3D \\
\hline Pictogramas & $X$ & $X$ & $X$ \\
\hline
\end{tabular}




\section{DESARROLLO DE LA ACTIVIDAD}

La experimentación tiene lugar a lo largo de dos días, el primer día dos sesiones, y el segundo una sesión aproximadamente, con la siguiente secuenciación de tiempos dependiendo de la edad:

Tabla 2. Secuenciación de la actividad en cada edad

\begin{tabular}{llll}
\hline \multicolumn{1}{c}{ Fases/Edad } & \multicolumn{1}{c}{ Edad 3 años } & \multicolumn{1}{c}{ Edad 4 años } & Edad 5 años \\
\hline Narración contexto & $1 / 2$ hora & $1 / 2$ hora & $1 / 2$ hora \\
\hline Asamblea & $10-15 \mathrm{~min}$ & $10-20 \mathrm{~min}$ & $20-30 \mathrm{~min}$ \\
\hline $\begin{array}{l}\text { Búsqueda del tesoro en pequeños } \\
\text { grupos }\end{array}$ & $10 \mathrm{~min} / \mathrm{grupo}$ & $8 \mathrm{~min} / \mathrm{grupo}$ & $5 \mathrm{~min} / \mathrm{grupo}$ \\
\hline $\begin{array}{l}\text { Representación del plano y puesta } \\
\text { en común }\end{array}$ & $20-30 \mathrm{~min}$ & $40 \mathrm{~min}$ & 50 minutos \\
\hline Trabajo con monedas & $40 \mathrm{~min}$ & $50 \mathrm{~min}$ & 50 min \\
\hline
\end{tabular}

\section{RESULTADOS}

A modo de ejemplo mostramos algunas de las fotografías de la implementación en 3 años (por cuestiones de privacidad, algunas de ellas aparecen borrosas).

En la primera fase, se realiza la lectura del cuento y posterior debate en asamblea, para pasar a la indagación de las "señales" que ayuden a encontrar el tesoro dando paso a una posterior búsqueda activa (figura 1). 
Figura 1. Comienzo de la actividad (lectura de cuento y búsqueda de pictogramas)
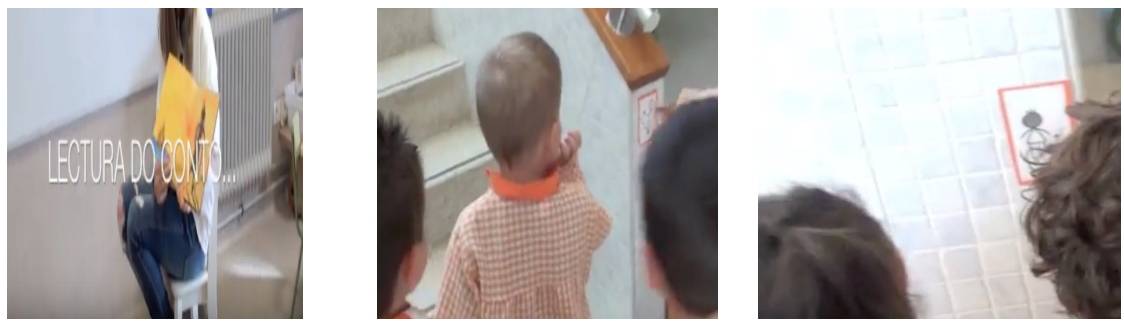

En la figura 2 vemos imágenes que hacen alusión a la búsqueda activa y al tesoro en sí (dependiendo del caso, pan de molde o monedas de chocolate, entre otros).

Figura 2. Búsqueda del tesoro de modo activo
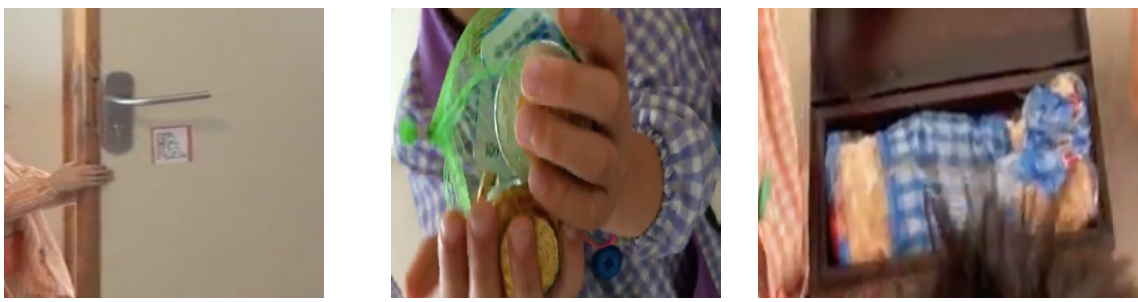

Finalmente, se pasa a la fase de formalización matemática, tanto a través de representación simbólica como de la comunicación oral (figura 3).

Figura 3. Trabajo posterior en clase con las representaciones
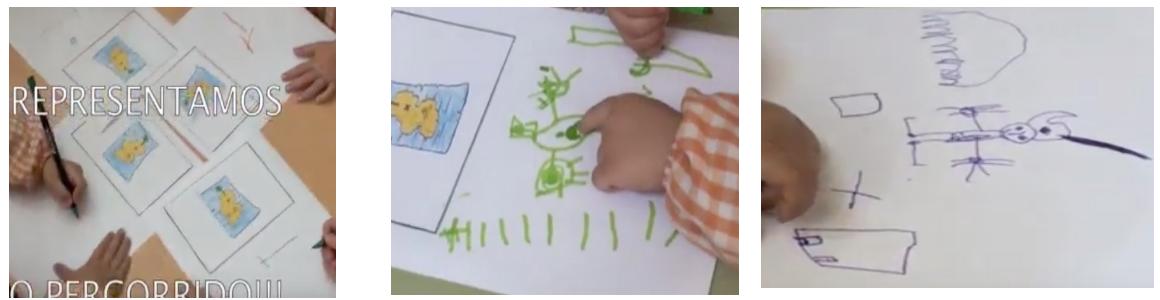

Algunas imágenes de la implementación en 5 años se pueden ver en Berciano et al. (2016). 


\section{DESCRIPCIÓN DE ALGUNAS DE LAS PRODUCCIONES POR EDADES}

En 3 años, a pesar de que aparecen diferentes representaciones, en todas ellas adquiere relevancia el tesoro como punto clave en el plano. Dependiendo del nivel cognitivo y los intereses mostrados a lo largo del itinerario el plano realizado muestra otros elementos con mayor o menor detalle.

Figura 4. Planos de recorridos, 3 años.
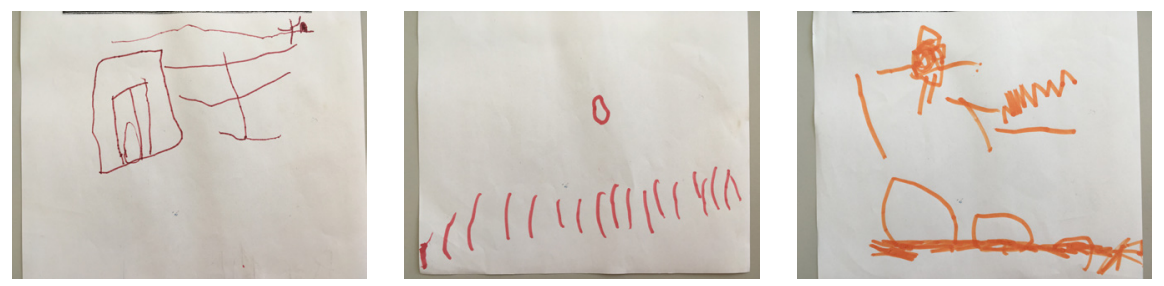

A los 4 años se aprecia una mayor concreción en aspectos relacionados con la capacidad de orientación. En concreto, el tesoro tiene una importancia relevante dentro de los dibujos pero comienzan a aparecer otros puntos de referencia singulares, (puerta, escaleras, árbol, ...) con un nivel de detalle diferente según el niño o la niña que lo realiza.

Figura 5. Planos de recorridos, 4 años.
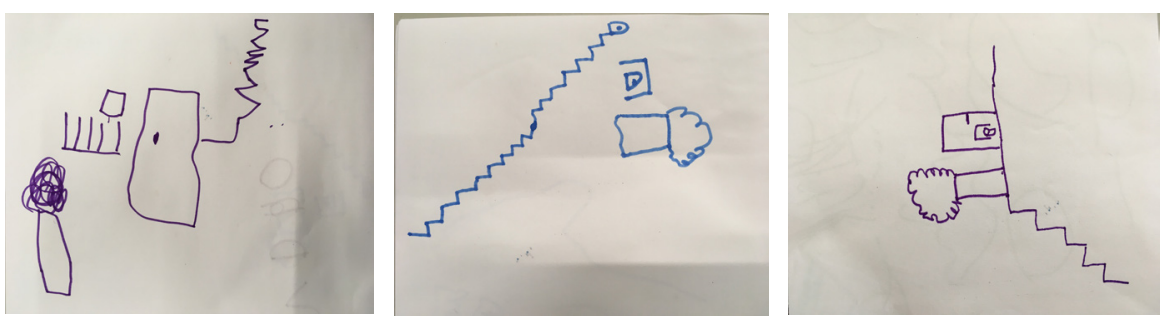

En las producciones de 5 años se observa como el tesoro no es el elemento protagonista de la representación, y los elementos singulares aparecen ordenados manteniendo una relación de orden que se corresponde con su localización real. 
Figura 6. Planos de recorridos, 5 años.
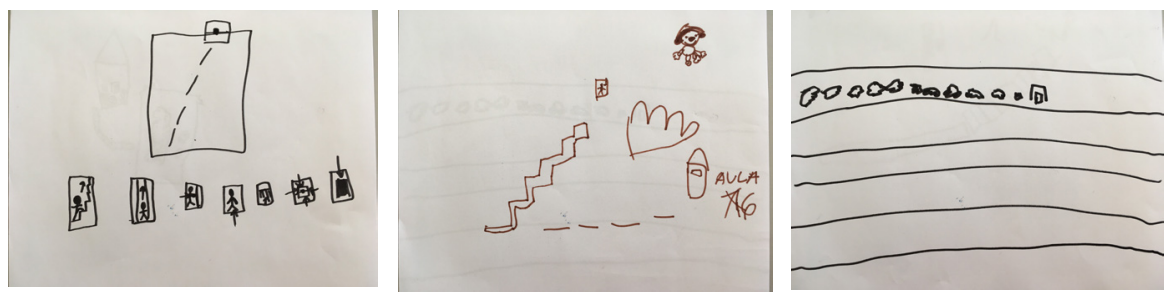

En definitiva, vemos que en edades 3 y 4 años la historia, la intriga, el contexto se antepone a la vivenciación del desarrollo de la búsqueda, por lo que parte del recorrido es omitido en sus representaciones y sólo se fijan en algunos puntos o elementos. En este sentido, algunos niños o niñas de 3 años se quedan sólo con el elemento final, mientras que en 4 años ya aparecen más partes del recorrido. Por el contrario, el alumnado de 5 años vive cada uno de los elementos del recorrido de la búsqueda del tesoro, y así queda registrado en sus planos, con detalles, simbología, ...

\section{DESCRIPCIÓN DE LAS DIFICULTADES DESDE EL PUNTO DE VISTA DOCENTE}

En este apartado queremos mostrar algunos de los aspectos más complicados de la experimentación docente llevada a cabo, con el fin de analizar su transferibilidad a otras escuelas.

Una de las complicaciones que tiene el realizar este tipo de actividad es que la planificación previa no garantiza que se puedan anticipar los resultados. Éstos, muchas veces, son impredecibles y esta incertidumbre aumenta cuanto más pequeños sean los niños. Se sabe de antemano que ante un rastreo, una salida del aula, $u$ otras actividades fuera de la rutina, el alumnado es receptivo, pero el grado de implicación y si van o no a comprender los mensajes, encontrar o no el tesoro,... esto no se sabe hasta la experimentación.

Para el caso del alumnado de menor edad, esta actividad incrementa su dificultad en el sentido de que, al ser una actividad grupal y debido a las características de su edad, los niños y las niñas no suelen desarrollar la actividad del modo esperado a priori. Siempre hay alumnos que toman la iniciativa y que tiran 
del grupo y otros que se dejan guiar, añadiendo un punto de complejidad a la hora de fomentar la participación de todos y todas. Sin embargo, a medida que aumenta la edad, se observa que las ideas se hablan y se llega a acuerdos de itinerarios que seguir, decisiones que tomar, etc.

Los resultados, en general, son muy difíciles de cuantificar y de calificar. La ausencia de producciones y registro de todas las decisiones tomadas para llegar al fin produce un vacío en la evaluación del proceso. Por otro lado, la observación de las producciones finales muestran algunos niveles de aprendizaje de los niños y las niñas, pero no recogen todo lo deseado. Esto se da sobre todo en 3 y 4 años donde la destreza asociada a la motricidad todavía no está muy desarrollada y un análisis de sus producciones refleja la omisión de muchos datos, aunque algunos de ellos no sean debidos al desconocimiento.

El trabajo en equipo en edades tempranas es difícil, por lo que hay tener en cuenta la conformación de los grupos de búsqueda de tesoro. Para que los equipos funcionen, resulta idóneo establecer roles de tal modo que se repartan obligaciones para el logro de un mismo objetivo.

Las conexiones: surge la dificultad para el docente de conectar la actividad con otras áreas (literatura y música) y en la misma área (orientación espacial, números, medida, cuantificadores, ...), para buscar la mayor realidad y significatividad de la misma.

Los tiempos: el tiempo de ejecución de la búsqueda no se puede anticipar con exactitud. Hay que estimar tiempos abiertos, de modo que cada grupo pueda explorar, observando, sin presiones... Hay que tener en cuenta que cuanto más corta es la edad, más tiempo se consume en la ejecución y en la toma de decisiones, por lo que hay que medir muy bien los tiempos.

Esta implementación se ha hecho en todo momento con dos docentes; para no solaparse la ejecución de la búsqueda de los grupos debe haber al menos dos docentes, una persona para acompañar, sin intervenir, al grupo que está realizando la actividad y otra que se quede en el aula con el resto del grupo-aula.

\section{DISCUSIÓN}

Los resultados obtenidos en esta práctica ponen de manifiesto el potencial de esta actividad, tanto a nivel metodológico como para el desarrollo cognitivo del alumnado. La metodología utilizada en el desarrollo del proceso de enseñanza-aprendizaje invita al alumnado a opinar, interactuar, comprobar y registrar y 
comunicar sus conclusiones con un lenguaje matemático lo más preciso posible (acorde con los principios de la EMR; Freudenthal, 1977).

La interacción con el espacio contextualiza un problema y lo hace real, lo que permite un aprendizaje significativo, con sentido y servicial para otras situaciones. En este sentido, la representación escrita cobra una especial importancia, debido a que los dibujos, trazos y registros de sus producciones están de acuerdo a la etapa evolutiva del niño o niña (acorde con Berciano et al., 2017). Sin embargo, también debemos saber que en ocasiones no consigue plasmar todas las ideas que éste o ésta verbaliza. A los 3 años hay más dificultad para recoger sus ideas a través del dibujo y trazos que con 5 años; de ahí la importancia de preguntar al niño o niña que explique sus producciones, para que pueda manifestar sus creencias e ideas al resto del grupo aula y profesorado.

El aprendizaje entre iguales respeta los ritmos individuales de desarrollo de los niños y niñas, y las aportaciones y discusiones crean pequeños conflictos que permiten avanzar en la construcción del conocimiento, y en particular de los planos y sus representaciones. Además, esta interacción entre iguales conlleva a una responsabilidad grupal, a formar parte de un todo, en el que el niño o la niña se apoya en el grupo, y no recae en él o ella toda la presión de la resolución con éxito de la actividad.

Por último, nos gustaría señalar que la información extraída de esta práctica puede ayudar a docentes a tomar decisiones mejores y más justificadas para el tratamiento de la orientación espacial en el aula de infantil, siempre y cuando el diseño de actividades cumplan las características que aquí destacamos: 1) a través de la búsqueda del tesoro se desarrollan de forma práctica y didáctica conceptos matemáticos abstractos que resultan confusos en edades tempranas; 2) la transversalidad que tiene esta actividad permite un alto nivel de contenidos diversos (educación para la paz, resolución de conflictos, respeto de las producciones,...); 3) la vivenciación provoca entusiasmo e interés por la realización de la actividad y los objetivos a lograr; 4) el ritmo individual de aprendizaje es respetado, debido a que conviven diferentes ritmos de madurez, en un entorno en el que todos tienen cabida y algo que aportar. Nadie se siente rechazado; 5) la evaluación individualiza a través del registro final individual del proceso de la actividad aporta una herramienta al profesorado para poder hacer juicios individuales de cada niño o niña con respecto a su capacidad de orientación. 


\section{REFERENCIAS}

Alsina, Á. (2009). El aprendizaje realista: una contribución de la investigación en Educación Matemática a la formación del profesorado. En M.J. González, M.T. González y J. Murillo (Eds.), Investigación en Educación Matemática XIII (pp. 119-127). Santander: SEIEM.

Berciano, A., Jiménez-Gestal, C. \& Salgado-Somoza, M. (2016). Tratamiento de la Orientación en el Aula de Educación Infantil desde la perspectiva de la Educación Matemática Realista, Números. Revista de Didáctica de la Matemática, 93, 31-43.

Berciano, A., Jiménez-Gestal, C. y Salgado, M. (2017). Kindergartners' Use of Symbols in the Semiotic Representation of 3-Dimensional Changes. International Electronic Journal of Mathematics Education, 11(4), 311-331.

Clements, D.H. \& Sarama, J. (2009). Learning and Teaching Early Math. The Learning Trajectories Approach. New York: Taylor \& Francis.

Freudenthal, H. (1977). Antwoord door prof. dr h. freudenthal na het verlenen van het eredoctoraat. (Respuesta del Prof. Dr H. Freudenthal al serle otorgado un doctorado honorario). Euclides, 52, 336-338.

Gonzato, M., Fernández Blanco, T. y Díaz Godino, J. (2011). Tareas para el desarrollo de habilidades de visualización y orientación espacial. Números, Revista de Didáctica de las Matemáticas, 77, 99-117

MEC (2007). ORDEN ECI/3960/2007, de 19 de diciembre, por la que se establece el currículo y se regula la ordenación de la educación infantil. BOE.

NTCM (2000). Principles and standards for school mathematics. Reston, Va.: The National Council of Teachers of Mathematics.

Rico, L. (1993). Mathematics assessment in the Spanish educational system. En M. Niss, Cases of Assessment in Mathematics Education (pp. 9-20). Dordrecht: Kluwer Academic Publishers.

CLARA JIMÉNEZ-GESTAL

Domicilio: Dpto. Matemáticas y Computación

Universidad de La Rioja

Complejo Científico y Tecnológico

Madre de Dios 53

26006 Logroño, España

Teléfono: $\quad+34941299461$ 